\title{
Ultimate energy recovery from spent relativistic electron beam in energy recovery linear accelerators
}

\author{
I. V. Konoplev®, ${ }^{1, *}$ Ya. Shashkov $\odot,{ }^{2}$ A. Bulygin $\odot,{ }^{2}$ M. A. Gusarova, ${ }^{2}$ and F. Marhauser ${ }^{3}$ \\ ${ }^{1}$ JAI, Department of Physics, University of Oxford, Oxford, OX1 3RH, United Kingdom \\ ${ }^{2}$ National Research Nuclear University MEPhI, Moscow, Russia \\ ${ }^{3}$ Thomas Jefferson National Accelerator Facility, Newport News, Virginia 23606, USA
}

(Received 13 March 2020; accepted 22 June 2020; published 7 July 2020)

\begin{abstract}
Energy recovery linear accelerators (ERLs) rely upon single-axis superconducting radio frequency (SRF) cavities to be an efficient source of relativistic electrons for high energy and nuclear physics. SRF cavities are also considered relevant for next-generation photon factories and radio-isotope production facilities. The ultimate energy recovery capability for the accelerator would be the ability to operate with a sufficiently spent beam and decrease the energy of the beam before the beam dump to a value lower than the beam injection energy. This is especially important for high current accelerators, where the beam injection energy could be as high as several $\mathrm{MeV}$, and systems where partial beam loss can be expected. The efficient operation of energy recovery in linear accelerators is adversely affected by the typical degradation of the beam quality and current loss. This hinders the application of the ERLs in research and industry, and enabling the use of spent (partially lost current), degraded beams should broaden their application. We suggest that the use of the asymmetric operating mode observed in the dual-axis asymmetric cavities will enable such ultimate capabilities. We discuss the advantages of the application of fields of different amplitude along the cavity accelerating and decelerating axes and demonstrate that the fields can be tuned separately in each axis of the dual-axis cavity. The design of such a cavity and ways to optimize the energy recovery of a spent electron beam by tuning the dual-axis asymmetric SRF cavity are discussed.
\end{abstract}

DOI: 10.1103/PhysRevAccelBeams.23.071601

\section{INTRODUCTION}

The concept of an asymmetric, dual-axis superconducting radio frequency (SRF) cavity (Fig. 1) has recently been proposed followed by preliminary studies of the electromagnetic properties of such a structure [1-4]. Dual-axis (also named twin-axis) cavities for particle acceleration have been considered before [5,6], and it has been recently suggested that dual-axis, asymmetric cavities can increase the beam breakup (BBU) instability threshold current $[7,8]$. We note that in all the previous studies the design of the cavity assures a flat and identical operating field profile along the axis of both sections (symmetric operating mode). The JLab team in collaboration with the team from Old Dominion University have built and successfully tested the first niobium, single-cell, twin-axis SRF cavity $[9,10]$.

To improve the energy efficiency of the energy recovery linac (ERL), SRF cavities are used. After transportation and

\footnotetext{
*Corresponding author. ivan.konoplev@physics.ox.ac.uk

Published by the American Physical Society under the terms of the Creative Commons Attribution 4.0 International license. Further distribution of this work must maintain attribution to the author(s) and the published article's title, journal citation, and DOI.
}

interaction with various targets, the electron beam current loss and its quality degradation, i.e., the emittance increases in the transverse and longitudinal phase space, will be observed. Even in the absence of interactions with collimators or targets, the beam may experience current loss (space-charge repulsion), and its quality will degrade due to coherent synchrotron radiation affecting the efficiency of the energy recovery [11-21]. As a result, state-of-the-art ERL design efforts focus on the preservation of the beam current and quality [21-23]. Furthermore, despite the deceleration and electron beam energy reduction, the electron beam is disposed in a dump (beam collector) carrying a significant amount of energy. Typical ERL injection energies range from 1 to $10 \mathrm{MeV}$, and current ERL designs cannot reduce the beam energy below that value. In conventional ERLs (average current, tens of $\mathrm{mA}$ ), the energy at the dump is typically larger than the energy of the injected beam, and the power dumped to the collector can range from $100 \mathrm{~kW}$ to $1 \mathrm{MW}$. It is clear that the reduction of this value can be beneficial [23], and it is especially important if an average ERL beam current of $1 \mathrm{~A}$ is realized, as proposed, for instance, in Ref. [1]. In this case, the power carried by the decelerated electron beam at the collector can be of the order of several megawatts, and the load on the beam collector becomes a significant issue even if the electron energy at the dump is the same as the 

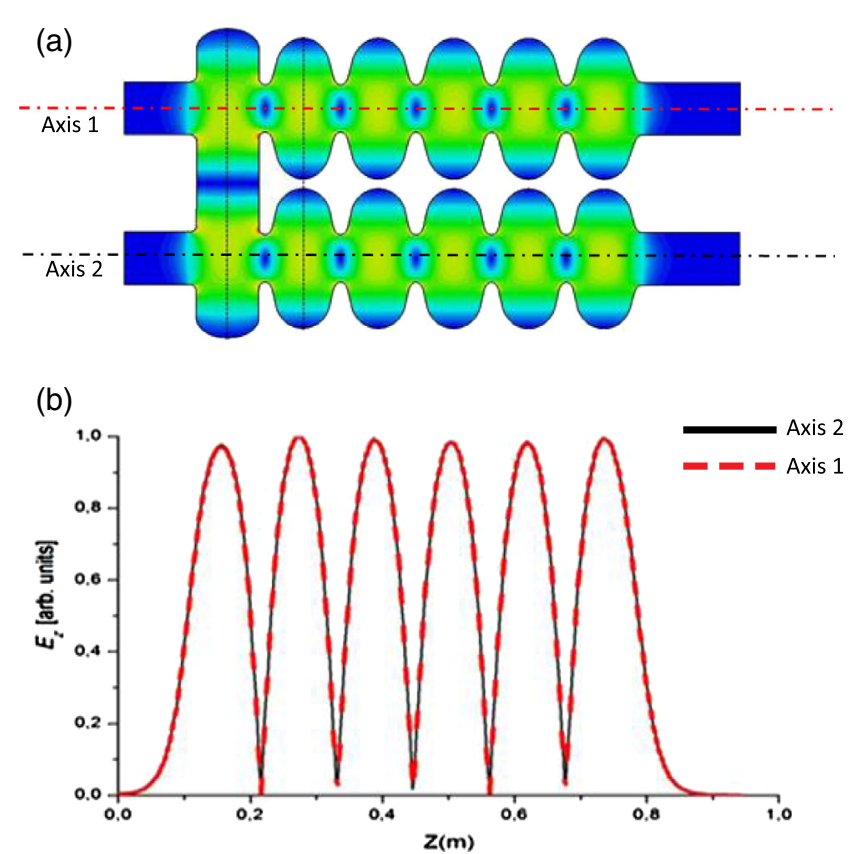

FIG. 1. (a) A schematic of the 11-cell rf cavity with a contour plot of the $E_{z}$ field component (accelerating field) of the operating mode; (b) the dependence of the absolute value of the $E_{z}$ field (operating mode) on the longitudinal coordinate $z$. The corresponding relationships are shown for both axes of the cavity (solid and dashed lines).

injected beam energy. This means that a reduction of the beam energy at the beam dump is vital to reduce the size and complexity of the beam collector while mitigating radiation and material activation concerns. The capabilities to recover energy from the spent beam and reduce the energy of the beam at the dump will broaden the use of the high current ERLs, making them attractive for medical and industrial applications.

In this work, a change from the paradigm of using flat identical fields along both acceleration and deceleration passes of the beams is proposed. We suggest to use a dualaxis cavity, previously proposed to increase the BBU start current via localization of the higher-order modes (HOMs), to exhibit the asymmetric operating mode which has different accelerating and decelerating field profiles. The asymmetry of the mode should enable efficient energy recovery from the degraded spent electron beam and to decrease the beam energy at the dump below the injected beam energy. Here, we discuss the limitations of the conventional approach and suggest using a decelerating field with a higher decelerating voltage than the accelerating voltage but not too high to avoid the beam reflection inside the cavity. We demonstrate the feasibility of such an ERL based on a dual-axis, multicell asymmetric cavity design and show the independent tunability of the fields along each axis, which can also be beneficial for further improvement of the ERL performance.

\section{CHALLENGES OF THE CONVENTIONAL ENERGY RECOVERY APPROACH AND POSSIBLE SOLUTIONS}

In a conventional, single-axis ERL cavity, the accelerating and decelerating electron beam bunches propagate along the same axis, experiencing identical amplitudes of the electric rf fields. The accelerated and decelerated bunches are separated by an odd number of half rf wavelengths allowing (theoretically) for an energy recovery of up to $100 \%$. The original design of an asymmetric dual-axis cavity [1-4] attempted to replicate this scenario. A crosssectional view of the original $1.3 \mathrm{GHz}$ dual-axis, multicell cavity (numerical model) is depicted in Fig. 1(a). This figure also shows a contour plot of the electric fields of the operating $\mathrm{TM}_{010} \pi$ mode employed for acceleration (axis 1) and deceleration (axis 2). The longitudinal components of the normalized electric field amplitudes, $E_{z}$, along the accelerating and decelerating axes are shown in Fig. 1(b), and in this case the field profiles are identical along both axes. The accelerating and decelerating (A/D) five-cell structures are coupled by a resonant bridging cell at the operating frequency. It is important to note that identical field patterns along both axes can be achieved by proper dimensional or frequency tuning of midcells and end cells, though the cavity cells in the A/D structure can be of different shape [1]. In this work, the numerical studies were carried out using the CST studio suite (CST) code [26].

In reality, however, the degradation of the beam quality, e.g., due to energy loss and interactions with targets has to be taken into account. As a result, the ability to tailor the field experienced by the decelerated beam independently from that experienced by the accelerating beam becomes important. To realize such an asymmetric operating mode, a dual-axis asymmetric cavity [1], which previously has been considered to increase BBU instability start current via localization of the HOMs should be considered. While in previous works the field patterns (amplitudes and structures) of the operating mode were the same along both axes (symmetric mode), here we suggest to reconsider the structure of the dual-axis cavity to observe the excitation of the asymmetric operating mode. The use of two types of cell contours, specifically, Tesla-type (T) cells [Fig. 2(a)] [1-3] and low-loss-type (L) cells [Fig. 2(b)] [21], is proposed. The choice of these cells was prompted by the fact that their properties and dimensions are well known $[21,24,25]$, while the general applicability of the approach is not affected. We assume that the $\mathrm{L}$ and $\mathrm{T}$ cells are located along axis 1 and axis 2 of the cavity [Fig. 3(a)], respectively. The differing on-axis field patterns for the $\mathrm{TM}_{010} \pi$ mode are shown in Fig. 3(b). The field amplitude along the decelerating axis (solid black line) is on average around $20 \%$ higher than the field amplitude along the accelerating axis (dashed red line) at the same energy stored in the cavity, and the field flatness along each five-cell structure is $99 \%$. 

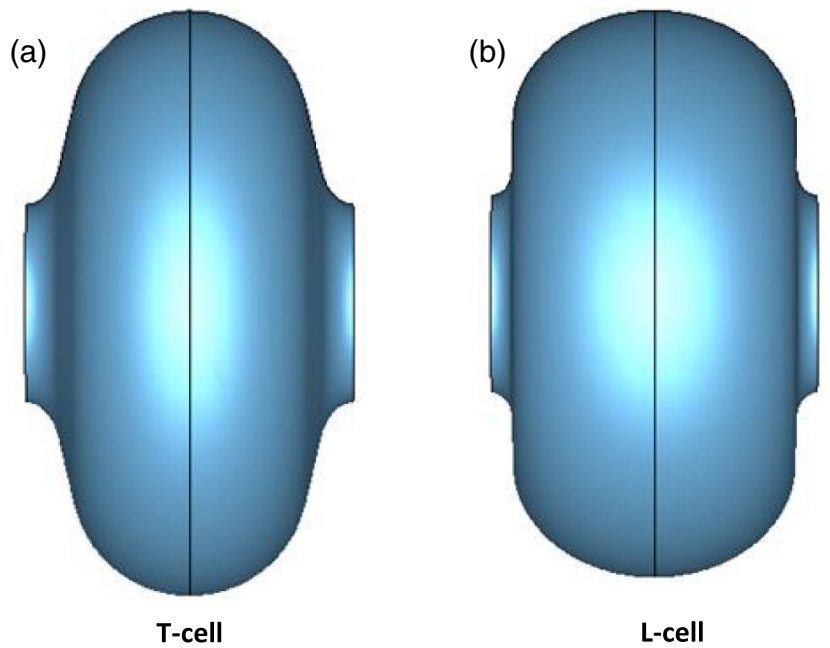

FIG. 2. 3D drawings of (a) the elliptical or Tesla-type (T) cell and (b) the low loss (L) cell.

To illustrate and discuss the results observed, let us assume a single-mode ideal bunch approximation and investigate the effect of the operating field and limits of the energy recovery. The ideal bunch means that all electrons are in a single point, while if the bunch has

(a)

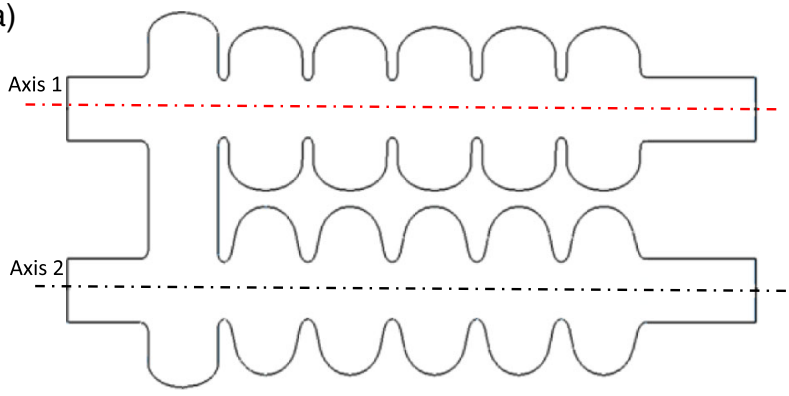

(b)

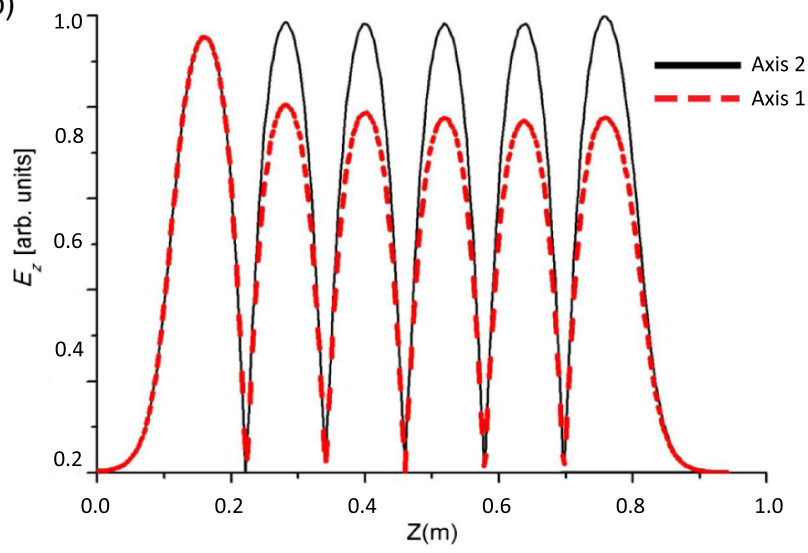

FIG. 3. (a) A schematic of the dual-axis asymmetric cavity constructed with the $\mathrm{T}$ (axis 2) and $\mathrm{L}$ (axis 1) cells connected by the racetrack bridge cell ( $\mathrm{T}$ and $\mathrm{L}$ configuration). (b) The dependence of the absolute value of the accelerating field $\left(E_{z}\right)$ along the longitudinal coordinate $z$ on both axes. finite dimensions, electrons are distributed in some rf phase interval. The energy exchange between the ideal bunch and the electromagnetic fields inside the cavity, along each of the axes, leads to a change in the beam energy. At the exit of a cavity, the beam energy can be estimated by

$$
W^{1,2}=W_{0}^{1,2} \pm c \int_{0}^{L / c} q^{1,2}(t) \beta_{z}^{1,2}(t) E_{z}^{1,2}(t) d t
$$

where $\beta_{z}^{1,2}$ and $E_{z}^{1,2}$ are the normalized longitudinal beam velocities and the electric fields, respectively, shown in Figs. 1(b), 3(b), and 4(b). The $q(t)=n(t) e$ is the total charge, $c$ is the speed of light, superscript indices 1 and 2 indicate the

(a)
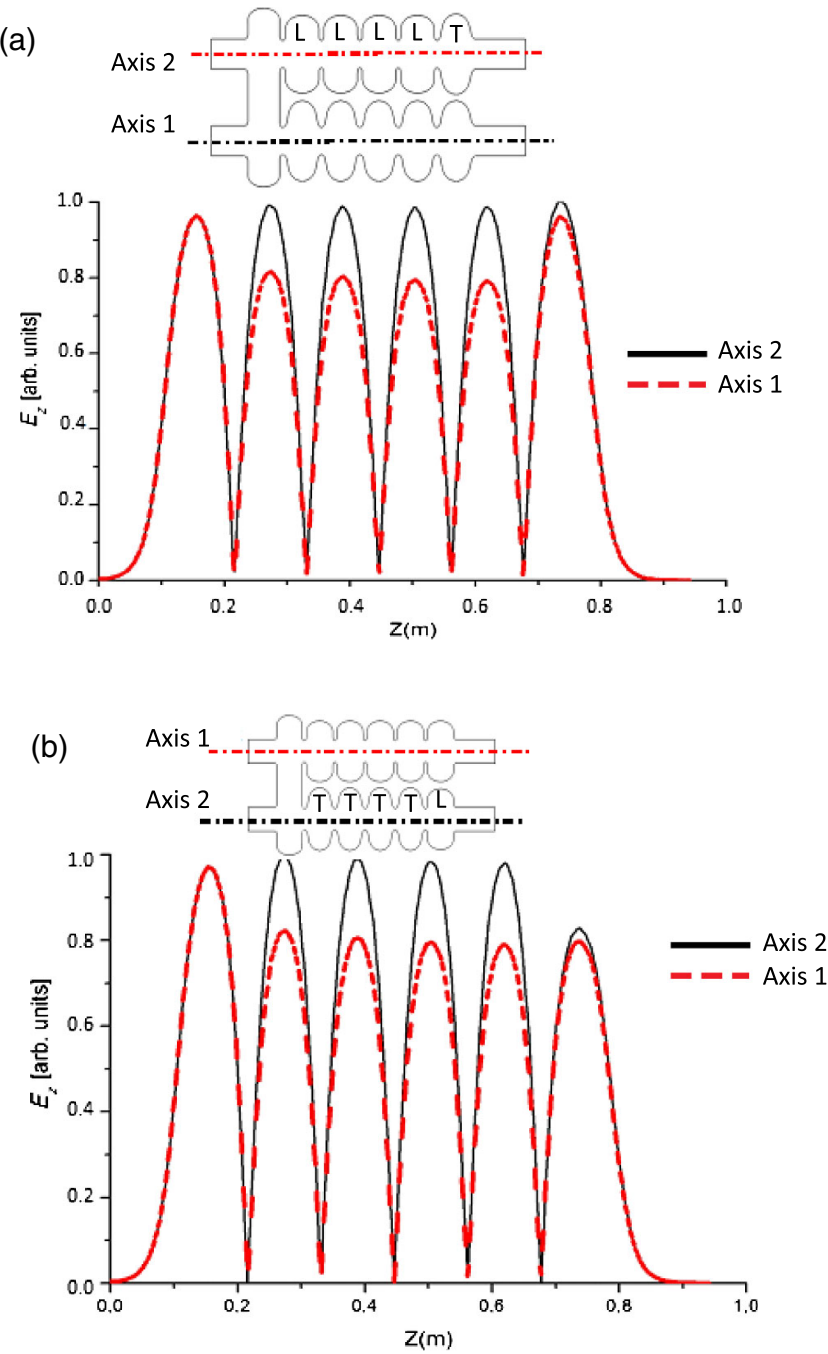

FIG. 4. (a) The dependence of the absolute value of the accelerating field $\left(E_{z}\right)$ along the longitudinal coordinate $z$ on both axes if the dual-axis asymmetric cavity is constructed with the $\mathrm{T}$ (axis 2) and $\mathrm{L}+\mathrm{T}$ (axis 2) cavity cells as shown in the inset. (b) The variation of the absolute value of the accelerating field $\left(E_{z}\right)$ along the longitudinal coordinate $z$ for both axes. This is in the case that the dual-axis asymmetric cavity is constructed with the $\mathrm{L}$ (axis 1) and $\mathrm{T}+\mathrm{L}$ (axis 2) cells as shown in the inset. 
accelerating (index 1) and the decelerating (index 2) axis, $L$ is the length of the cavity, and $W_{0}^{1,2}$ are the energies of the beams injected into the cavity; the "+" takes into account energy gain and "-" energy recovery. If the ideal bunch approximation is not applied, $q^{1,2}(t) \beta_{z}^{1,2}(t) E_{z}^{1,2}(t)$ should be substituted with $\left\langle q^{1,2}\left(t, \theta_{0}^{1,2}\right) \beta_{z}^{1,2}\left(t, \theta_{0}^{1,2}\right) E_{z}^{1,2}\left(t, \theta_{0}^{1,2}\right)\right\rangle_{\theta_{0}}$, where brackets indicate the averaging over $\theta_{0}^{1,2}$ (distribution along the rf phase interval $)$ and $q^{1,2}\left(t, \theta_{0}^{1,2}\right), \beta_{z}^{1,2}\left(t, \theta_{0}^{1,2}\right)$, and $E_{z}^{1,2}\left(t, \theta_{0}^{1,2}\right)$ are the bunch longitudinal profiles, velocities distribution, and fields experienced by each individual particle along the axes, respectively. For the short and finite length bunches, the following inequalities take place: $q^{1,2}(t) \beta_{z}^{1,2}(t) E_{z}^{1,2}(t)>$ $\left\langle q^{1,2}\left(t, \theta_{0}^{1,2}\right) \beta_{z}^{1,2}\left(t, \theta_{0}^{1,2}\right) E_{z}^{1,2}\left(t, \theta_{0}^{1,2}\right)\right\rangle_{\theta_{0}}$ and, if the ideal bunches are phased correctly with respect to acceleration or deceleration potentials, the expression (1) gives a maximum and minimum for $W^{1}$ and $W^{2}$, respectively. Here the ideal bunch approximation will be used to identify the ERL limits, and $W^{2}$-considering the deceleration in all cells-is the beam energy at the beam dump. For convenience, and without loss of generality, we describe the beam degradation by an energy variation term $\delta W$, which is a function of the longitudinal momentum $\left(\beta_{z}=p_{z} c / W\right)$. During beam transportation, for instance, some of the longitudinal momentum can be transferred into transverse momentum or is lost during collisions or due to radiation, thus resulting in the term $\delta W$. Assuming also that at the injection point and at the dump $\beta_{z} \sim 1$, one can rewrite the energy at the dump as

$$
W^{2} \cong W_{0}^{1}-\delta W+c \int_{0}^{L / c} q^{1}(t) \beta_{z}^{1}(t) E_{z}^{1}(t)(1-\eta(t)) d t,
$$

where $\eta(t)=q^{2}(t) \beta_{z}^{2}(t) E_{z}^{2}(t) / q^{1}(t) \beta_{z}^{1}(t) E_{z}^{1}(t)$ is the ratio which, in conventional ERLs $\left[E_{z}^{1}(t)=E_{z}^{2}(t)\right]$ is less than 1 as $q^{1}(t) \beta_{z}^{1}(t)>q^{2}(t) \beta_{z}^{2}(t)$ due to beam degradation $(\delta W \neq 0)$. If $\delta W / W_{0}^{1} \ll 1$ (conventional ERLs) but not zero, one gets

$$
W^{2} / W_{0}^{1} \cong 1+c \int_{0}^{L / c} \mu(t)(1-\eta(t)) d t>1,
$$

where $\mu(t)=q^{1}(t) \beta_{z}^{1}(t) E_{z}^{1}(t) / W_{0}^{1}$. This illustrates that in conventional ERLs $W^{2}>W_{0}^{1}$ and only in the ideal case, i.e., $E_{z}^{1}(t)=E_{z}^{2}(t)$ and $\delta W=0$, one gets $W^{2}=W_{0}^{1}$. If, on the other hand, the asymmetric dual-axis cavity is used with $E_{z}^{1}(t) \neq E_{z}^{2}(t)$ as shown in Fig. 3(b), this leads to $W^{2} / W_{0}^{1}<1$ as the integral becomes negative [according to Eq. (3)], showing that more energy can be extracted from the beam. Consequently, the beam energy at the dump is below the energy of the injected beam $W_{0}^{1}$. In particular, Fig. 3(b) demonstrates one of the possible cases where $E_{z}^{1}>E_{z}^{2}$, allowing for compensation of the beam degradation including the partial loss of the beam charge $q$. An additional advantage is the increase in the energy acceptance, which is proportional to the field amplitude, thus accepting a beam with a higher energy spread [27-30] or/and with the dephased from the optimal position bunches as compared with the single-axis cavities.

\section{DESIGN OF THE CAVITY FOR MAXIMAL ENERGY RECOVERY}

The ability to design the field pattern on one of the axes independently from the other is ideal in order to tailor a dual-axis cavity for a specific machine. There are a number of viable methods through which this can be achieved. A modification of just the last cells in the cavities is a possibility. This is shown in the insets in Figs. 4(a) and 4(b), where the $\mathrm{L}$ cell has been substituted with a $\mathrm{T}$ cell on axis 1 [Fig. 4(a)] and the $\mathrm{T}$ cell with an $\mathrm{L}$ cell on axis 2 [Fig. 4(b)]. The resulting field patterns are plotted along each axis for each case. In the first case [Fig. 4(a)], the field amplitude in the last, modified cell along axis 1 is higher as compared with other cells, while the field flatness along axis 2 is preserved in the five-cell structure. In the second case [Fig. 4(b)], the field amplitude in the last, modified cell along axis 2 in decreased, while the field flatness along axis 1 in the five-cell structure is not affected. In both cases, the rf fields are mainly altered locally in the modified cell, allowing the effective accelerating or decelerating voltage to be accurately controlled. It is important to note that the substitution of the last cells did not lead to a change of the operating mode frequency, since the frequencies of the $\mathrm{L}$ and $\mathrm{T}$ cells are similar by design. We also note that the modification of additional cells, if necessary, will allow a
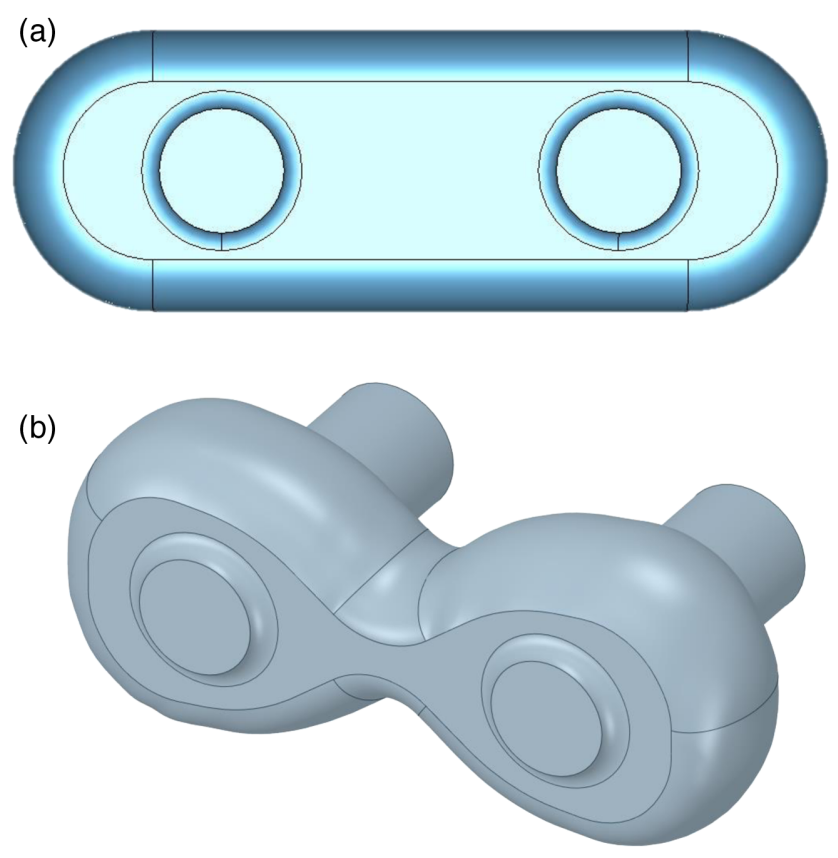

FIG. 5. 3D illustrations of the original racetrack coupling bridge: (a) front view and (b) view of the modified coupling bridge. 
TABLE I. Comparison of the parameters of the $\mathrm{T}$ and $\mathrm{L}+\mathrm{T}$ cavity with the original and the new coupling bridges.

\begin{tabular}{lcc}
\hline \hline & Old bridge & New bridge \\
\hline Operating mode frequency $(\mathrm{GHz})$ & 1.300027 & 1.300027 \\
Nearest mode frequency $(\mathrm{GHz})$ & 1.299311 & 1.299418 \\
$E_{p} / E_{a}$ & 2.71 & 2.28 \\
$B_{p} / E_{a}(\mathrm{mT} / \mathrm{MV} / \mathrm{m})$ & 6.11 & 4.43 \\
$R / Q(\mathrm{Ohm})$ axis 1 & 380 & 406 \\
$R / Q(\mathrm{Ohm})$ axis 2 & 289 & 273 \\
$V_{z}(\mathrm{MV})$ axis $1^{\mathrm{a}}$ & 1.76 & 1.82 \\
$V_{z}(\mathrm{MV})$ axis $2^{\mathrm{a}}$ & 1.53 & 1.49 \\
$R / Q(\mathrm{Ohm})$-axis 1 nearest mode & 203 & 196 \\
$R / Q(\mathrm{Ohm})$-axis 2 nearest mode & 281 & 338 \\
$G,(\mathrm{Ohm})$-operating mode & 276.8 & 277.2 \\
\hline \hline
\end{tabular}

${ }^{\mathrm{a}}$ At $1 \mathrm{~J}$ stored energy

smoother tapering of either the accelerating or decelerating fields, which will improve the energy recovery efficiency achieved by the system.

To further improve the SRF cavity design, the peak rf fields on the cavity surface located within the bridge cell have been minimized via dimensional optimizations. The original bridge cell had a racetrack geometry [Fig. 5(a)], and the ratios of the peak magnetic flux density $B_{p}$ and peak electric field $E_{p}$ to the accelerating field $E_{a}$ were relatively high, compared to those achievable in conventional cavities [9,22-25]. High fields could potentially limit the achievable accelerating gradients in the cavity and increase the chances of field emission. Furthermore, transverse fields along the axes need to be minimized in order to mitigate the deflection (kick) of the beam when traversing the bridge cavity. These issues have been addressed in the design of the twin-axis, single-cell cavity design developed at JLab $[9,10]$ by choosing a figure-eight cell contour. In the present work, this contour has been adopted as a starting point for further optimization of the bridge cell. rf models of the original racetrack and a new bridge cell design are shown Fig. 5, and Table I lists the corresponding parameters of the cavities with the original and modified bridge cell. Comparing the parameters, we note a significant reduction of $E_{p} / E_{a}$ from 2.71 to 2.28 and of $B_{p} / E_{a}$ from 6.1 to 4.43 . This values are close to or better than the values obtainable in conventional SRF cavities $([9,21-25]$.

\section{CONCLUSION}

Research presented here has demonstrated that the dualaxis asymmetric SRF cavity can support accelerating and decelerating fields of different amplitudes. The possibilities of operating with an electron beam of lower quality and less current and to reduce the beam power at the beam collector are the major advantages of the proposed system. The results show that the use of such a structure will be beneficial for ERL by recovering energy from a spent and degraded quality beam. Bringing the beam energy at the collector below the injected energy values by having the decelerating potential higher as compared with accelerating potential [Fig. 3(b)] does not mean, in general, breaking the energy balance between accelerating and decelerating sections. The spent beam current, for example, may be less than that of the injected beam and/or beam phase spread may affect the efficiency of the energy recovery. If the balance is broken, and energy extracted from the beam is above the energy beam yields in the acceleration section, to maintain the rf energy balance, additional $\mathrm{rf}$ couplers will be required to transfer the excess of the energy to a load. Clearly, such rf energy transfer systems may be complex and have to be specially designed for each specific case. An example of such a load can be the SRF gun. The SRF gun based on a $1.3 \mathrm{GHz}$ Tesla-like cell [Fig. 2(a)] has recently been developed and tested by HZDR (Germany) and JLab (USA) teams [31], and the possibility of positioning the injector and accelerating module in a single cryostat has been discussed [32] by the JLab team. Taking into account results shown in Refs. [31,32], one may consider coupling the SRF injector and the dual-axis asymmetric cavity as shown in Fig. 6. Figure 6(a) shows the schematic and the contour plot of the asymmetric operating field, while Fig. 6(b) shows the dependences of the operating field amplitudes on the longitudinal coordinate along the axes. The dashed line shows the SRF injector and A/D structure merging plane and beam injected and final energies are also shown. To operate such a system, i.e., to maintain the energy balance between accelerating and decelerating sections, the decelerating field amplitude should be higher as compared with the accelerating field. Also, there is no need to design additional rf couplers to unload the rf power excess from decelerating section of the A/D structure. Achieving low energy at the beam dump and conducting efficient energy recovery from the spent beam will make ERLs attractive for a wider range of research and industrial applications [11-17].

We suggested that the dual-axis cavity should use cells of different geometries to realize the asymmetric operating mode, and we demonstrated that the voltage experienced by a beam on the deceleration axis can be larger than that on the accelerating axis. The independent adjustment of the field pattern of the operating mode along each axis has also been demonstrated. By extension, the results illustrate the possibility of field pattern tailoring (by other means) so that the field's amplitudes gradually change from cell to cell, leading to the field tapering, though that would require a modification of more than a single cell, as studied herein. We have also modified the original $\mathrm{rf}$ design of the cavity to improve its reliability by replacing the original racetrack cell with a figure-eight bridge cell to minimize the surface peak fields at a given accelerating fields. The capability of the dual-axis cavity concept with a variable asymmetric operating mode can be particularly 


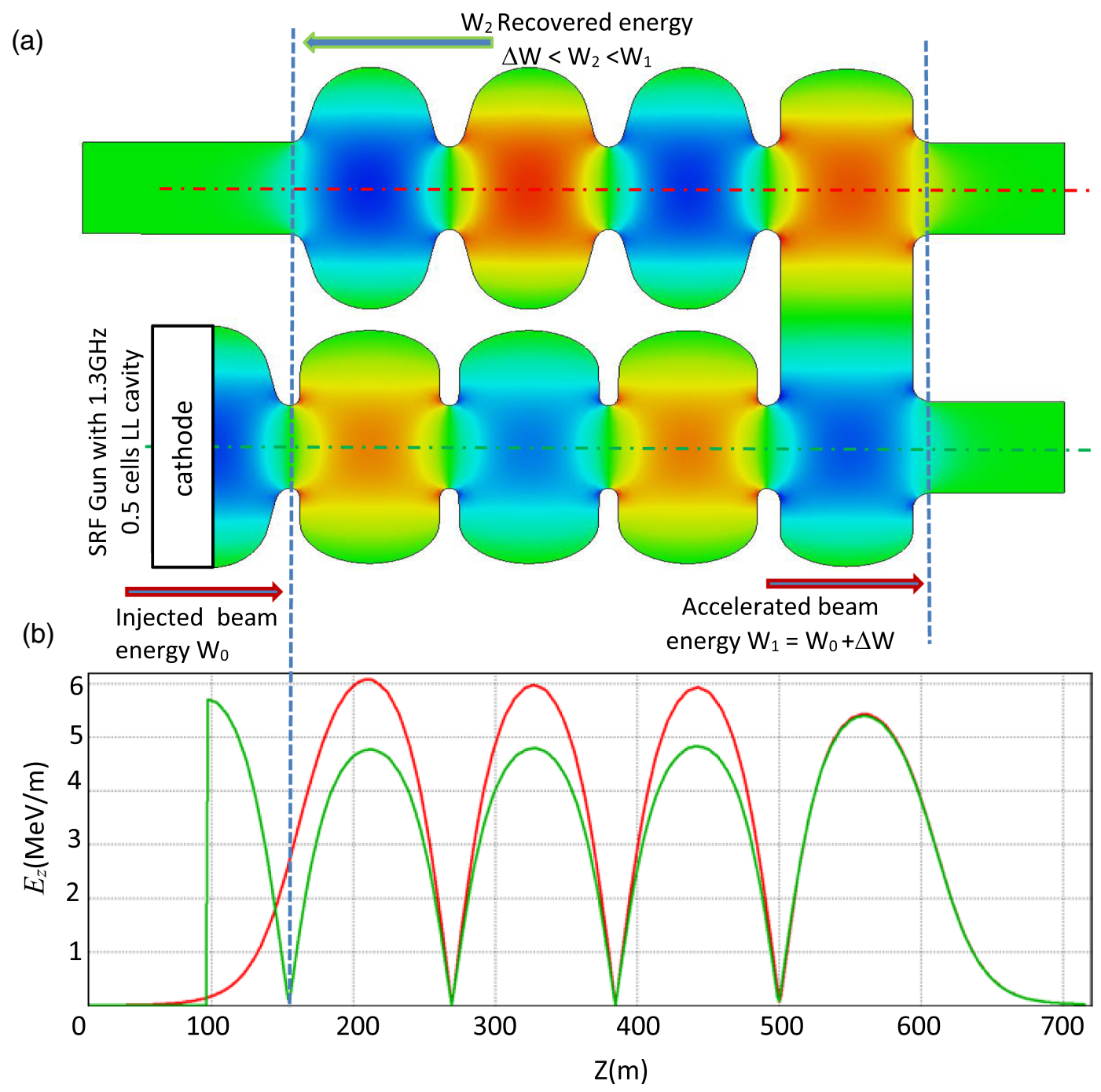

FIG. 6. (a) Schematic of the SRF injector and SRF cavity and contour plot of the operating field. (b) The dependence of the absolute value of the accelerating field $\left(E_{z}\right)$ along the longitudinal coordinate $z$ on both axes and comparison of the dependence with the schematic shown in (a).

beneficial for high-energy ERLs, which may exhibit significant energy losses due to coherent synchrotron radiation. The result of the studies should be similarly beneficial for ERLs conceived for radioisotope and $\mathrm{THz}$ radiation production with prebunched electron beams and where beam current losses are expected [11-17]. The findings should contribute toward the development of future high-current ERLs for a broad range of industrial, medical, and research applications.

\section{ACKNOWLEDGMENTS}

The authors are very grateful to Professor A. Seryi for useful and stimulating discussions. The authors thank STFC UK for their support through the STFC UKRI Grants No. ST/M003590/1, No. ST/P000967/1, No. ST/ S000224/1, and No. ST/S000046/1. I. K. thanks the Royal Society for their support via Grant No. EC\R3\170010.
We also thank the Russian Foundation for Basic Research for support of the work via the RFBR research Project No. 18-32-00990.

[1] R. Ainsworth, G. Burt, I. V. Konoplev, and A. Seryi, Asymmetric dual axis energy recovery linac for ultrahigh flux sources of coherent $\mathrm{x}$-ray and $\mathrm{THz}$ radiation: Investigations towards its ultimate performance, Phys. Rev. Accel. Beams 19, 083502 (2016).

[2] I. V. Konoplev, K. Metodiev, A. J. Lancaster, G. Burt, R. Ainsworth, and A. Seryi, Experimental studies of 7-cell dual axis asymmetric cavity for energy recovery linac, Phys. Rev. Accel. Beams 20, 103501 (2017).

[3] A. Seryi, Ultrahigh flux compact x-ray source, Patent PCT Application No. PCT/GB2012/052632, 2012; R. Ainsworth, G. Burt, I. V. Konoplev, and A. Seryi, 
Asymmetric superconducting rf structure, Patent PCT Application No. PCT/GB2015/053565, 2015.

[4] M. Topp-Mugglestone, I. V. Konoplev, and A. Seryi, Studies of high order modes in asymmetric dual-axis cavity, Appl. Phys. Lett. 113, 243503 (2018).

[5] C.-x. Wang, J. Noonan, and J. W. Lewellen, Dual-axis energyrecovery linac, in Proceedings of ERL07, Daresbury, UK, 2007, p. 18, https://accelconf.web.cern.ch/erl07/papers/18 .pdf.

[6] S. Noguchi and E. Kako, Multi-beam accelerating structures, in Proceedings of SRF 2003, Lubeck, Germany (2003), p. 317

[7] G. H. Hoffstaetter and I. V. Bazarov, Beam-breakup instability theory for energy recovery linacs, Phys. Rev. Accel. Beams 7, 054401 (2004).

[8] G. H. Hoffstaetter, I. V. Bazarov, and C. Song, Recirculating beam-breakup thresholds for polarized higher-order modes with optical coupling, Phys. Rev. Accel. Beams 10, 044401 (2007).

[9] S. U. De Silva, H. Park, J. R. Delayen, F. Marhauser, and A. Hutton, Electromagnetic design of a superconducting twin axis cavity, in Proceedings of LINAC2016, East Lansing, MI (2016), MOPLR030, https://doi.org/ 10.18429/JACoW-LINAC2016-MOPLR030; H. Park, F. Marhauser, A. Hutton, S. U. De Silva, and J. R. Delayen, Development of a superconducting twin axis cavity, in Proceedings of LINAC2016, East Lansing, MI (2016), THPLR037, https://doi.org/10.18429/JACoW-LINAC2016 -THPLR037.

[10] A. Hutton and H. Areti, Accelerator stewardship test facility program-Elliptical twin cavity for accelerator applications, JLab Report No. JLAB-HEP15-03, 2015, https://www.osti.gov/biblio/1209532.

[11] D. H. Bilderback, J. D. Brock, D. S. Dale, K. D. Finkelstein, M. A. Pfeifer, and S. M. Gruner, Energy recovery linac (ERL) coherent hard X-ray sources, New J. Phys. 12, 035011 (2010); S. M. Gruner, Energy recovery linacs as synchrotron radiation sources, Rev. Sci. Instrum. 73, 1402 (2002).

[12] D. Pellegrini, A. Latina, D. Schulte, and S. Alex, Bogacz, Beam-dynamics driven design of the LHeC energyrecovery linac, Phys. Rev. Accel. Beams 18, 121004 (2015).

[13] C. Montag, G. Bassi, J. Beebe-Wang, J. S. Berg, M. Blaskiewicz, A. Blednykh, J. M. Brennan, S. Brooks, K. A. Brown, K. A. Drees et al., eRHIC design status, in Proceedings of IPAC2018, Vancouver, BC, Canada (JACoW Publishing, 2019), pp. 628-631, https://accelconf .web.cern.ch/ipac2018/papers/tuygbd3.pdf; Y. Jing, Y. Hao, and V. N. Litvinenko, Compensating effect of the coherent synchrotron radiation in bunch compressors, Phys. Rev. Accel. Beams 16, 060704 (2013).

[14] H. Zhang, I. V. Konoplev, G. Doucas, and J. Smith, Concept of a tunable source of coherent $\mathrm{THz}$ radiation driven by a plasma modulated electron beam, Phys. Plasmas 25, 043111 (2018).

[15] S. Antipov, C. Jing, M. Fedurin, W. Gai, A. Kanareykin, K. Kusche, P. Schoessow, V. Yakimenko, and A. Zholents, Experimental Observation of Energy Modulation in Electron Beams Passing through Terahertz Dielectric Wakefield Structures, Phys. Rev. Lett. 108, 144801 (2012).
[16] P. Piot, Y.-E. Sun, T. J. Maxwell, J. Ruan, A. H. Lumpkin, M. M. Rihaoui, and R. Thurman-Keup, Observation of coherently enhanced tunable narrow-band terahertz transition radiation from a relativistic sub-picosecond electron bunch train, Appl. Phys. Lett. 98, 261501 (2011).

[17] S. Antipov, M. Babzien, C. Jing, M. Fedurin, W. Gai, A. Kanareykin, K. Kusche, V. Yakimenko, and A. Zholents, Subpicosecond Bunch Train Production for a Tunable mJ Level THz Source, Phys. Rev. Lett. 111, 134802 (2013).

[18] V. N. Starovoitova, L. Tchelidze, and D. P. Wells, Production of medical radioisotopes with linear accelerators, Appl. Radiat. Isot. 85, 39 (2014).

[19] D. A. Rotscha, M. A. Brown, J. A. Nolen, T. Brossard, W. F. Henning, S. D. Chemerisov, R. G. Gromov, and J. Greene, Electron linear accelerator production and purification of scandium-47 from titanium dioxide targets, Appl. Radiat. Isot. 131, 77 (2018).

[20] Y. Ekinci, T. Garvey, A. Streun, A. Wrulich, and L. Rivkin A high-brightness accelerator-based EUV source for metrology applications, in Photomask Technology 2018, SPIE Proceedings Vol. 10810, 108100W (SPIE-International Society for Optical Engineering, Bellingham, WA, 2018), https://doi.org/10.1117/12.2501930.

[21] D. Angal-Kalinin et al., PERLE. Powerful energy recovery linac for experiments. Conceptual design report, J. Phys. G 45, 065003 (2018).

[22] A. Khan, O. Boine-Frankenheim, F. Hug, and C. Stoll, Beam matching with space charge in energy recovery linacs, Nucl. Instrum. Methods Phys. Res., Sect. A 948, 162822 (2019).

[23] I. Ben-Zvi, Superconducting energy recovery linacs, Supercond. Sci. Technol. 29, 103002 (2016).

[24] H. Padamsee, J. Knobloch, and T. Hays, RF Superconductivity for Accelerators, Wiley Series in Beam Physics and Accelerator Technology (Wiley, New York, 1998).

[25] J. Sekutovicz et al., Cavities for JLab's $12 \mathrm{GeV}$ upgrade, in Proceedings of the 2003 Particle Accelerator Conference, Portland, OR (IEEE, New York, 2003) p. 1395-1397; V. Shemelin, Optimal choice of cell geometry for a multicell superconducting cavity, Phys. Rev. Accel. Beams 12, 114701 (2009).

[26] CST Microwave Studio, SIMULIA, Dassault Systems, http://www.3ds.com.

[27] V. L. Bratman, A. D. R. Phelps, and A. V. Savilov, Recovery of electron energy in cyclotron autoresonance masers, Phys. Plasmas 4, 2285 (1997).

[28] T. J. Orzeehowski, B. R. Anderson, J. C. Clark, W. M. Fawley, A. C. Paul, D. Prosnitz, E. T. Scharlemann, S. M. Yarema, D. B. Hopkins, A. M. Sessler, and J. S. Wurtele, High-Efficiency Extraction of Microwave Radiation from a Tapered-Wiggler Free-Electron Laser, Phys. Rev. Lett. 57, 2172 (1986).

[29] P. Sprangle, C.-M. Tang, and W. M. Manheirner, Nonlinear theory of free-electron lasers anti efficiency enhancement, Phys. Rev. A 21, 302 (1980).

[30] C. Emma, N. Sudar, P. Musumeci, A. Urbanowicz, and C. Pellegrini, High efficiency tapered free-electron lasers with a prebunched electron beam, Phys. Rev. Accel. Beams 20, 110701 (2017). 
[31] A. Arnold et al., Fabrication, tuning, treatment and testing of two 3.5 cell photo-injector cavities for the ELBE linac, in Proceedings of the 15th International Conference on RF Superconductivity, 2011, Chicago (2011), http://cds.cern .ch/record/1436389/files/EuCARD-CON-2011-047.pdf? version=1; RF performance results of the 2nd ELBE SRF gun, in Proceedings of the 17th International Conference on RF Superconductivity, 2015, Whistler, British Columbia, Canada (2015), https://accelconf.web.cern.ch/srf2015/ papers/thpb055.pdf; R. Xiang et al., SRF gun and SRF linac driven $\mathrm{THz}$ at ELBE successfully in user operation, in Proceedings of the 19th International Conference on RF Superconductivity (SRF 2019), Dresden, Germany (JACoW Publishing, 2019), p. 917-919, https://doi.org/ 10.18429/JACoW-SRF2019-THP032.

[32] K. E. Deitrick, G. A. Krafft, B. Terzić, and J. R. Delayen, High-brilliance, high-flux compact inverse Compton light source, Phys. Rev. Accel. Beams 21, 080703 (2018). 